\title{
A study on a new and fast fixed point iteration process in Banach spaces
}

\author{
Kadri Dogan \\ Department of Computer Engineering, Artvin Coruh University, Artvin, Turkey \\ Received: 6 February 2019, Accepted: 19 February 2020 \\ Published online: 18 March 2020.
}

\begin{abstract}
In this study, it is proposed a new general fixed point iteration method for approximation of fixed point of contraction mappings in Banach spaces. Next, it has been proven that this iteration method is faster than the AK, S-Picard, Thakur, Vatan twosteps fixed point iterative methods recently described in the literature. Moreover, it is shown that the convergence of Mann fixed point iteration method is equivalent to the convergence of newly defined fixed point iteration method. Also, numerical examples are given to support the analytic proofs. Finally, the data dependence of this fixed point iteration method has been proven.
\end{abstract}

Keywords: Fixed point iterative method, strong convergence, the rate of convergence, Banach spaces, data dependency.

\section{Introduction and preliminaries}

The fixed point theory is well-known in the current literature because it provides useful tools for solving many problems with applications in the different fields such as engineering, chemistry, game theory and economics. However, once we have decided on the existence of a fixed point of a certain mapping, finding the fixed point of mapping is not an easy task, so we use iterative process to calculate of them. Then, it is necessary to develop an iterative process which approximate the solution of nonlinear equations that has a good rate of convergence. Over the years, many researchers have efforts to develop iterative processes having faster the rate of convergence than others. In this direction, some of notable studies were conducted by Mann, Ishikawa, Noor, Suantai, Karakaya, Gursoy, Dogan, Yildirim, Karahan, Sainuan, Agarwal, Rhoades and Khan [1-17]. In addition, the fixed point mappings were studied as much as studies on the iterative methods. Different varieties of these mappings are available in the literature. The well known of them are contraction mappings, nonexpansive mappings and generalizations of them. Therefore, in this study, a new fixed point iterative method (7) is constructed and well-known contraction mapping has been studied such that

$$
\|T x-T y\| \leq \delta\|x-y\|
$$

for $\delta \in(0,1)$.

In 1953, Mann [4] introduced a new iterative method known as Mann iterative method to approximate fixed point as follows:

$$
\left\{\begin{array}{l}
m_{0} \in C \\
m_{n+1}=\left(1-\varsigma_{n}\right) m_{n}+\varsigma_{n} T m_{n}, \text { for all } n \in[0, \infty)
\end{array}\right.
$$

In 2018, Dogan and Karakaya [22] introduced the following new iterative method known as S-Picard iterative method to

\footnotetext{
* Corresponding author e-mail: dogankadri@ hotmail.com
} 
approximate fixed point of contraction maps:

$$
\left\{\begin{array}{l}
k_{0} \in C \text { initial point } \\
k_{n+1}=\left(1-\varsigma_{n}\right) T r_{n}+\varsigma_{n} T d_{n} \\
d_{n}=\left(1-\tau_{n}\right) T k_{n}+\tau_{n} T r_{n} \\
r_{n}=T k_{n}, \text { for all } n \in[0, \infty)
\end{array}\right.
$$

In 2016, Karakaya et al. [21] introduced the following iterative process known as Vatan two-step iteration process to approximate fixed point of $T$, defined by:

$$
\left\{\begin{array}{l}
v_{0} \in C \text { initial point } \\
v_{n+1}=T\left(\left(1-\varsigma_{n}\right) k_{n}+\varsigma_{n} T\left(k_{n}\right)\right) \\
k_{n}=T\left(\left(1-\tau_{n}\right) v_{n}+\tau_{n} T\left(v_{n}\right)\right), \text { for all } n \in[0, \infty)
\end{array}\right.
$$

Thakur et al. (2016) used a new iterative process termed Thakur iterative method for approximation of fixed points, defined by:

$$
\left\{\begin{array}{l}
k_{0} \in C \text { initial point } \\
w_{n}=\left(1-\varsigma_{n}\right) u_{n}+\varsigma_{n} T u_{n} \\
v_{n}=T\left(\left(1-\tau_{n}\right) u_{n}+\tau_{n} w_{n}\right) \\
u_{n+1}=T\left(v_{n}\right), \text { for all } n \in[0, \infty)
\end{array}\right.
$$

Recently, Ullah and Arshad [24] introduced the following new iterative method termed AK iteration process to approximate fixed point, defined by:

$$
\left\{\begin{array}{l}
x_{0} \in C \text { initial point } \\
z_{n}=T\left(\left(1-\varsigma_{n}\right) x_{n}+\varsigma_{n} T x_{n}\right) \\
y_{n}=T\left(\left(1-\tau_{n}\right) z_{n}+\tau_{n} T z_{n}\right) \\
x_{n+1}=T\left(y_{n}\right), \text { for all } n \in[0, \infty)
\end{array}\right.
$$

When the iteration methods described above are discussed in this article, it is seen that there is a race among researchers to identify the fastest iteration method. This race has made important contributions to the fixed point theory. For this reason, it was defined the following iteration method in this study.

$$
\left\{\begin{array}{l}
\rho_{0} \in C \text { initial condition } \\
\rho_{n+1}=\left(\prod_{i=1}^{m} T\right)\left(\left(1-\varsigma_{n}\right) \omega_{n}+\varsigma_{n} T\left(\omega_{n}\right)\right) \\
\omega_{n}=\left(\prod_{j=1}^{p} T\right)\left(\left(1-\tau_{n}\right) \varkappa_{n}+\tau_{n} T\left(\varkappa_{n}\right)\right) \\
\varkappa_{n}=\left(\prod_{k=1}^{t} T\right)\left(\rho_{n}\right), \text { for all } n \in \mathbb{N} .
\end{array}\right.
$$

where $\varsigma_{n}, \tau_{n} \in[0,1], T(\rho) \times T(\rho)=T(T(\rho))$ and $m, p, t \in \mathbb{N}$.

In this iteration method, different iteration methods can be obtained by giving value to the representations of $\mathrm{m}$, $\mathrm{p}$ and $\mathrm{t}$. The iteration method at the lowest rate of convergence of these iteration methods corresponds to the speed of the AK iteration method known as the fastest three-step iteration method in the literature. Therefore, the new iteration method has an important status in terms of speed.

Definition 1. [25] Let $B$ be a Banach space and $T, \breve{T}: B \rightarrow B$ two operators. if

$$
\|T x-\check{T} x\| \leq \varepsilon
$$


for all $x \in B$ and some $\varepsilon>0$. Then $\breve{T}$ is an approximate operator of $T$

Theorem 1. [26] Let $B$ be a real Banach space, let $E \subset B$ be a nonempty convex and closed set, and let $\varepsilon>0$ be a fixed number. If $T: E \rightarrow E$ is a contractive operator with the fixed point a and $\check{T}: E \rightarrow E$ is an operator with a fixed point $b$, and the inequality (8) is hold. Then, we have

$$
\|a-b\| \leq \frac{\varepsilon}{1-\delta}
$$

Definition 2. [25] Let $\left\{a_{n}\right\}_{n=0}^{\infty}$ and $\left\{b_{n}\right\}_{n=0}^{\infty}$ be two sequences of real numbers which converge to a and b, respectively. Also, assume that

$$
\lim _{n \rightarrow \infty}\left|\frac{a_{n}-a}{b_{n}-b}\right|=l .
$$

In which case

- If $l=0$, then it is clear that $\left\{a_{n}\right\}_{n=0}^{\infty}$ converges faster to a than $\left\{b_{n}\right\}_{n=0}^{\infty}$ to $b$

- If $0<l<\infty$, then it is clear that $\left\{a_{n}\right\}_{n=0}^{\infty}$ and $\left\{b_{n}\right\}_{n=0}^{\infty}$ have the same rate of convergence.

Definition 3. [25] Let $\left\{v_{n}\right\}_{n=0}^{\infty}$ and $\left\{u_{n}\right\}_{n=0}^{\infty}$ be two fixed point iteration methods both converging to the same fixed point $\rho^{*}$ with the error estimates $\left\|v_{n}-p\right\| \leq a_{n}$ and $\left\|u_{n}-p\right\| \leq b_{n}$, where $\left\{a_{n}\right\}_{n=0}^{\infty}$ and $\left\{b_{n}\right\}_{n=0}^{\infty}$ be two sequences of real numbers converging to 0 . If $\left\{a_{n}\right\}_{n=0}^{\infty}$ converges faster than $\left\{b_{n}\right\}_{n=0}^{\infty}$, then $\left\{v_{n}\right\}_{n=0}^{\infty}$ converges faster than $\left\{u_{n}\right\}_{n=0}^{\infty}$ to $\rho^{*}$.

Lemma 1. [26] Let $\left\{a_{n}\right\}$ be a nonnegative sequence for which one supposes there exists $n_{0} \in \mathbb{N}$ such that for all $n \geq n_{0}$.

For $\varepsilon_{n} \in(0,1) \forall n \in \mathbb{N}, \sum_{n=1}^{\infty} \varepsilon_{n}=\infty$ and $b_{n} \geq 0$, let's assume that the inequality

$$
a_{n+1} \leq\left(1-\varepsilon_{n}\right) a_{n}+\varepsilon_{n} b_{n}
$$

is provided. Then

$$
0 \leq \lim _{n \rightarrow \infty} \sup a_{n} \leq \lim _{n \rightarrow \infty} \sup b_{n}
$$

Lemma 2. [20] Let $\left\{a_{n}\right\}_{n=0}^{\infty}$ and $\left\{b_{n}\right\}_{n=0}^{\infty}$ be nonnegative reel two sequences satisfiying the following inequality:

$$
a_{n+1} \leq\left(1-\varepsilon_{n}\right) a_{n}+b_{n}
$$

where $\varepsilon_{n} \in(0,1)$ for all $n \in \mathbb{N}, \sum_{n=0}^{\infty} \varsigma_{n}=\infty$ and $\frac{b_{n}}{\varepsilon_{n}} \rightarrow 0$ as $n \rightarrow \infty$, then $a_{n} \rightarrow 0$ as $n \rightarrow \infty$.

\section{Convergence Analysis}

Theorem 2. Let $E$ be a nonempty closed convex subset of a Banach space $B$ and $T: E \rightarrow E$ be a contraction mapping. Suppose that $\left\{\rho_{n}\right\}_{n=0}^{\infty}$ is an iterative sequence generated by (7) with $\left\{\varsigma_{n}\right\}_{n=0}^{\infty},\left\{\tau_{n}\right\}_{n=0}^{\infty} \subset[0,1]$ satisfying $\sum_{n=0}^{\infty} \tau_{n}=\infty$. Then the iterative process $\left\{\rho_{n}\right\}_{n=0}^{\infty}$ converges to a unique fixed point $\rho^{*}$ of $T$.

Proof. Theorem guaranteeing the existence and uniqueness of fixed point $\rho^{*}$ of contraction mapping is the well-known Banach contraction theorem. Thus, it will be shown that $\lim _{n \rightarrow \infty} \rho_{n}=\rho^{*}$. 
By using the iterative sequence (7) and the contraction mapping (1), we have

$$
\begin{aligned}
\left\|\rho_{n+1}-\rho^{*}\right\| & =\left\|\left(\prod_{i=1}^{m} T\right)\left(\left(1-\varsigma_{n}\right) \omega_{n}+\varsigma_{n} T\left(\omega_{n}\right)\right)-\rho^{*}\right\| \\
& \leq \delta^{m}\left\|\left(1-\varsigma_{n}\right) \omega_{n}+\varsigma_{n} T\left(\omega_{n}\right)-\rho^{*}\right\| \\
& \leq \delta^{m}\left(1-\varsigma_{n}\right)\left\|\omega_{n}-\rho^{*}\right\|+\delta^{m} \varsigma_{n}\left\|T\left(\omega_{n}\right)-\rho^{*}\right\| \\
& \leq \delta^{m}\left(1-\varsigma_{n}(1-\delta)\right)\left\|\omega_{n}-\rho^{*}\right\| \\
& \leq \delta^{m}\left(1-\varsigma_{n}(1-\delta)\right)\left\|\left(\prod_{j=1}^{p} T\right)\left(\left(1-\tau_{n}\right) \varkappa_{n}+\tau_{n} T\left(\varkappa_{n}\right)\right)-\rho^{*}\right\| \\
& \leq \delta^{m+p}\left(1-\varsigma_{n}(1-\delta)\right)\left\|\left(1-\tau_{n}\right) \varkappa_{n}+\tau_{n} T\left(\varkappa_{n}\right)-\rho^{*}\right\| \\
& \leq \delta^{m+p}\left(1-\varsigma_{n}(1-\delta)\right)\left(1-\tau_{n}\right)\left\|\varkappa_{n}-\rho^{*}\right\|+\delta^{m+p}\left(1-\varsigma_{n}(1-\delta)\right) \tau_{n}\left\|T\left(\varkappa_{n}\right)-\rho^{*}\right\| \\
& \leq \delta^{m+p}\left(1-\varsigma_{n}(1-\delta)\right)\left(1-\tau_{n}(1-\delta)\right)\left\|\varkappa_{n}-\rho^{*}\right\| \\
& \leq \delta^{m+p}\left(1-\varsigma_{n}(1-\delta)\right)\left(1-\tau_{n}(1-\delta)\right)\left\|\left(\prod_{k=1}^{t} T\right)\left(\rho_{n}\right)-\rho^{*}\right\| \\
& \leq \delta^{m+p+t}\left(1-\varsigma_{n}(1-\delta)\right)\left(1-\tau_{n}(1-\delta)\right)\left\|\rho_{n}-\rho^{*}\right\| .
\end{aligned}
$$

Hence, we have

$$
\left\|\rho_{n+1}-\rho^{*}\right\| \leq \delta^{m+p+t}\left(1-\varsigma_{n}(1-\delta)\right)\left(1-\tau_{n}(1-\delta)\right)\left\|\rho_{n}-\rho^{*}\right\|
$$

By using induction, we obtain

$$
\begin{aligned}
\left\|\rho_{n+1}-\rho^{*}\right\| & \leq\left(\delta^{m+p+t}\right)^{n+1} \prod_{i=0}^{n}\left[\left(1-\varsigma_{i}(1-\delta)\right)\left(1-\tau_{i}(1-\delta)\right)\right]\left\|\rho_{0}-\rho^{*}\right\| \\
& \leq\left(\delta^{m+p+t}\right)^{n+1} \prod_{i=0}^{n}\left(1-\tau_{n}(1-\delta)\right)\left\|\rho_{0}-\rho^{*}\right\| .
\end{aligned}
$$

By using the facts that $1-\tau_{i}(1-\delta)<1$ for $\delta \in(0,1), \tau_{i}, \varsigma_{i} \in[0,1]$ and $1-x<e^{-x}$ for $x \in[0,1]$, we have

$$
\left\|\rho_{n+1}-\rho^{*}\right\| \leq \frac{\left(\delta^{m+p+1}\right)^{n+1}\left\|\rho_{0}-\rho^{*}\right\|}{e^{(1-\delta) \sum_{i=0}^{n} \tau_{i}}}
$$

Taking into account of the inequality (11), the following result can be easily reached.

$$
\lim _{n \rightarrow \infty} \rho_{n}=\rho^{*}
$$

Consequently, the iterative process (7) converges to a unique fixed point $\rho^{*}$ of $T$.

Theorem 3. Let $E$ be a nonempty closed convex subset of a Banach space $B$ and $T: E \rightarrow E$ be a contraction mapping. Suppose that $\left\{\rho_{n}\right\}_{n=0}^{\infty}$ and $\left\{m_{n}\right\}_{n=0}^{\infty}$ are two iterative sequences, which have the same fixed point $\rho^{*}$, defined by (7) and (2), respectively, such that $\left\{\varsigma_{n}\right\}_{n=0}^{\infty},\left\{\tau_{n}\right\}_{n=0}^{\infty} \subset[0,1]$ satisfying $\sum_{n=0}^{\infty} \tau_{i} \varsigma_{i}=\infty$. In which case, The following statements are verified.

(i) The iterative process $\left\{\rho_{n}\right\}_{n=0}^{\infty}$ converges to a unique fixed point $\rho^{*}$ of $T$,

(ii) The Mann iterative process $\left\{m_{n}\right\}_{n=0}^{\infty}$ converges to a unique fixed point $\rho^{*}$ of $T$.

Proof. $(i) \Rightarrow(i i)$ : Let's assume that the iterative method $\left\{\rho_{n}\right\}_{n=0}^{\infty}$ converges to a unique fixed point $\rho^{*}$ of $T$. Then, it will be proved that $\lim _{n \rightarrow \infty} m_{n}=\rho^{*}$. 
By using iterative processes (7), (2) and the contraction mapping (1), it is obtained

$$
\begin{aligned}
& \left\|\rho_{n+1}-m_{n+1}\right\|=\left\|\left(\prod_{i=1}^{m} T\right)\left(\left(1-\varsigma_{n}\right) \omega_{n}+\varsigma_{n} T\left(\omega_{n}\right)\right)-\left(1-\varsigma_{n}\right) m_{n}-\varsigma_{n} T m_{n}\right\| \\
& \leq\left\|\begin{array}{c}
\left(\prod_{i=1}^{m} T\right)\left(\left(1-\varsigma_{n}\right) \omega_{n}+\varsigma_{n} T\left(\omega_{n}\right)\right)-\left(\prod_{i=1}^{m-1} T\right)\left(\left(1-\varsigma_{n}\right) \omega_{n}+\varsigma_{n} T\left(\omega_{n}\right)\right) \\
+\left(\prod_{i=1}^{m-1} T\right)\left(\left(1-\varsigma_{n}\right) \omega_{n}+\varsigma_{n} T\left(\omega_{n}\right)\right)-\left(1-\varsigma_{n}\right) m_{n}-\varsigma_{n} T m_{n}
\end{array}\right\| \\
& \leq\left\|\left(\prod_{i=1}^{m} T\right)\left(\left(1-\varsigma_{n}\right) \omega_{n}+\varsigma_{n} T\left(\omega_{n}\right)\right)-\left(\prod_{i=1}^{m-1} T\right)\left(\left(1-\varsigma_{n}\right) \omega_{n}+\varsigma_{n} T\left(\omega_{n}\right)\right)\right\| \\
& +\left\|\left(\prod_{i=1}^{m-1} T\right)\left(\left(1-\varsigma_{n}\right) \omega_{n}+\varsigma_{n} T\left(\omega_{n}\right)\right)-\left(1-\varsigma_{n}\right) m_{n}-\varsigma_{n} T m_{n}\right\| \\
& \leq \delta^{m-1}\left[\left(1-\varsigma_{n}\right)\left\|T\left(\omega_{n}\right)-\omega_{n}\right\|+\left\|T\left(\left(1-\varsigma_{n}\right) \omega_{n}+\varsigma_{n} T\left(\omega_{n}\right)\right)-T\left(\omega_{n}\right)\right\|\right] \\
& +\left\|\left(\prod_{i=1}^{m-1} T\right)\left(\left(1-\varsigma_{n}\right) \omega_{n}+\varsigma_{n} T\left(\omega_{n}\right)\right)-\left(1-\varsigma_{n}\right) m_{n}-\varsigma_{n} T m_{n}\right\| \\
& \leq \delta^{m-1}\left(1-\varsigma_{n}(1-\delta)\right)\left\|T\left(\omega_{n}\right)-\omega_{n}\right\|+\left\|\left(\prod_{i=1}^{m-1} T\right)\left(\left(1-\varsigma_{n}\right) \omega_{n}+\varsigma_{n} T\left(\omega_{n}\right)\right)-\left(1-\varsigma_{n}\right) m_{n}-\varsigma_{n} T m_{n}\right\| \\
& \leq\left(\delta^{m-1}+\delta^{m-2}+\delta^{m-3}+\ldots+\delta^{2}+\delta\right)\left(1-\varsigma_{n}(1-\delta)\right)\left\|T \omega_{n}-\omega_{n}\right\| \\
& +\left\|T\left(\left(1-\varsigma_{n}\right) T\left(\varkappa_{n}\right)+\varsigma_{n} T\left(\omega_{n}\right)\right)-\left(1-\varsigma_{n}\right) m_{n}-\varsigma_{n} T m_{n}\right\| \\
& \leq\left(\delta^{m-1}+\delta^{m-2}+\delta^{m-3}+\ldots+\delta^{2}+\delta\right)\left(1-\varsigma_{n}(1-\delta)\right)\left\|T \omega_{n}-\omega_{n}\right\| \\
& +\left\|T\left(\left(1-\varsigma_{n}\right) \omega_{n}+\varsigma_{n} T\left(\omega_{n}\right)\right)-\left(1-\varsigma_{n}\right) \omega_{n}-\varsigma_{n} T\left(\omega_{n}\right)+\left(1-\varsigma_{n}\right) \omega_{n}+\varsigma_{n} T\left(\omega_{n}\right)-\left(1-\varsigma_{n}\right) m_{n}-\varsigma_{n} T m_{n}\right\| \\
& \leq\left(\delta^{m-1}+\delta^{m-2}+\delta^{m-3}+\ldots+\delta^{2}+\delta\right)\left(1-\varsigma_{n}(1-\delta)\right)\left\|T \omega_{n}-\omega_{n}\right\| \\
& +\left\|T\left(\left(1-\varsigma_{n}\right) \omega_{n}+\varsigma_{n} T\left(\omega_{n}\right)\right)-\left(1-\varsigma_{n}\right) \omega_{n}-\varsigma_{n} T\left(\omega_{n}\right)\right\|+\left\|\left(1-\varsigma_{n}\right) \omega_{n}+\varsigma_{n} T\left(\omega_{n}\right)-\left(1-\varsigma_{n}\right) m_{n}-\varsigma_{n} T m_{n}\right\| \\
& \leq\left(\delta^{m-1}+\delta^{m-2}+\delta^{m-3}+\ldots+\delta^{2}+\delta\right)\left(1-\varsigma_{n}(1-\delta)\right)\left\|T \omega_{n}-\omega_{n}\right\| \\
& +\left(1-\varsigma_{n}\right)\left\|T\left(\left(1-\varsigma_{n}\right) \omega_{n}+\varsigma_{n} T\left(\omega_{n}\right)\right)-\omega_{n}\right\|+\varsigma_{n}^{2} \delta\left\|T\left(\omega_{n}\right)-\omega_{n}\right\| \\
& +\left(1-\varsigma_{n}\right)\left\|\omega_{n}-m_{n}\right\|+\varsigma_{n}\left\|T\left(\omega_{n}\right)-T m_{n}\right\| \\
& \leq\left(\delta^{m-1}+\delta^{m-2}+\delta^{m-3}+\ldots+\delta^{2}+\delta+1\right)\left(1-\varsigma_{n}(1-\delta)\right)\left\|T \omega_{n}-\omega_{n}\right\| \\
& +\left(1-\varsigma_{n}(1-\delta)\right)\left\|\omega_{n}-m_{n}\right\| \text {. }
\end{aligned}
$$

Again, from iterative processes (7), (2) and the contraction mapping (1) we have

$$
\begin{aligned}
\left\|\varkappa_{n}-m_{n}\right\| & \leq\left\|\prod_{k=1}^{t} T\left(\rho_{n}\right)-\prod_{k=1}^{t-1} T\left(\rho_{n}\right)+\prod_{k=1}^{t-1} T\left(\rho_{n}\right)-m_{n}\right\| \\
& \leq\left\|\prod_{k=1}^{t} T\left(\rho_{n}\right)-\prod_{k=1}^{t-1} T\left(\rho_{n}\right)\right\|+\left\|\prod_{k=1}^{t-1} T\left(\rho_{n}\right)-m_{n}\right\|
\end{aligned}
$$




$$
\begin{aligned}
& \leq \delta^{t-1}\left\|T\left(\rho_{n}\right)-\rho_{n}\right\|+\left\|\prod_{k=1}^{t-1} T\left(\rho_{n}\right)-\prod_{k=1}^{t-2} T\left(\rho_{n}\right)+\prod_{k=1}^{t-2} T\left(\rho_{n}\right)-m_{n}\right\| \\
& \leq \delta^{t-1}\left\|T\left(\rho_{n}\right)-\rho_{n}\right\|+\left\|\prod_{k=1}^{t-1} T\left(\rho_{n}\right)-\prod_{k=1}^{t-2} T\left(\rho_{n}\right)\right\|+\left\|\prod_{k=1}^{t-2} T\left(\rho_{n}\right)-m_{n}\right\| \| \\
& \leq \delta^{t-1}\left\|T\left(\rho_{n}\right)-\rho_{n}\right\|+\delta^{t-2}\left\|T\left(\rho_{n}\right)-\rho_{n}\right\|+\left\|\prod_{k=1}^{t-2} T\left(\rho_{n}\right)-m_{n}\right\| \\
& \vdots \\
& \leq \delta^{t-1}\left\|T\left(\rho_{n}\right)-\rho_{n}\right\|+\delta^{t-2}\left\|T\left(\rho_{n}\right)-\rho_{n}\right\|+\delta^{t-3}\left\|T\left(\rho_{n}\right)-\rho_{n}\right\| \\
& +\delta^{t-4}\left\|T\left(\rho_{n}\right)-\rho_{n}\right\|+\ldots+\delta\left\|T\left(\rho_{n}\right)-\rho_{n}\right\|+\left\|T\left(\rho_{n}\right)-m_{n}\right\| \\
& \leq\left(\delta^{t-1}+\delta^{t-2}+\delta^{t-3}+\ldots+\delta+1\right)\left\|T\left(\rho_{n}\right)-\rho_{n}\right\|+\left\|\rho_{n}-m_{n}\right\| .
\end{aligned}
$$

Similarly

$$
\begin{aligned}
\left\|\omega_{n}-m_{n}\right\| & =\left\|\left(\prod_{j=1}^{p} T\right)\left(\left(1-\tau_{n}\right) \varkappa_{n}+\tau_{n} T\left(\varkappa_{n}\right)\right)-m_{n}\right\| \\
\leq & \left\|\left(\prod_{j=1}^{p} T\right)\left(\left(1-\tau_{n}\right) \varkappa_{n}+\tau_{n} T\left(\varkappa_{n}\right)\right)-\left(\prod_{j=1}^{p-1} T\right)\left(\left(1-\tau_{n}\right) \varkappa_{n}+\tau_{n} T\left(\varkappa_{n}\right)\right)\right\| \\
& +\left\|\left(\prod_{j=1}^{p-1} T\right)\left(\left(1-\tau_{n}\right) \varkappa_{n}+\tau_{n} T\left(\varkappa_{n}\right)\right)-m_{n}\right\| \\
& \leq\left(\delta^{p-1}+\delta^{p-2}\right)\left(1-\tau_{n}(1-\delta)\right)\left\|T \varkappa_{n}-\varkappa_{n}\right\|+\left\|\left(\prod_{j=1}^{p-2} T\right)\left(\left(1-\tau_{n}\right) \varkappa_{n}+\tau_{n} T\left(\varkappa_{n}\right)\right)-m_{n}\right\| \\
& \leq\left(\delta^{p-1}+\delta^{p-2}+\delta^{p-3}\right)\left(1-\tau_{n}(1-\delta)\right)\left\|T \varkappa_{n}-\varkappa_{n}\right\|+\left\|\left(\prod_{j=1}^{p-3} T\right)\left(\left(1-\tau_{n}\right) \varkappa_{n}+\tau_{n} T\left(\varkappa_{n}\right)\right)-m_{n}\right\| \\
& \vdots \\
& \leq\left(\delta^{p-1}+\delta^{p-2}+\delta^{p-3}+\ldots+\delta\right)\left(1-\tau_{n}(1-\delta)\right)\left\|T \varkappa_{n}-\varkappa_{n}\right\|+\left\|T\left(\left(1-\tau_{n}\right) \varkappa_{n}+\tau_{n} T\left(\varkappa_{n}\right)\right)-m_{n}\right\| \\
& \leq\left[\left(\delta^{p-1}+\delta^{p-2}+\delta^{p-3}+\ldots+\delta\right)\left(1-\tau_{n}(1-\delta)\right)+\left(\delta \tau_{n}+1\right)\right]\left\|T \varkappa_{n}-\varkappa_{n}\right\|+\left\|\varkappa_{n}-m_{n}\right\| .
\end{aligned}
$$

Substituting the inequality (13) in the inequality (14) we obtain

$$
\begin{aligned}
\left\|\omega_{n}-m_{n}\right\| & \leq\left[\left(\delta^{p-1}+\delta^{p-2}+\delta^{p-3}+\ldots+\delta\right)\left(1-\tau_{n}(1-\delta)\right)+\left(\delta \tau_{n}+1\right)\right]\left\|T \varkappa_{n}-\varkappa_{n}\right\| \\
& +\left(\delta^{t-1}+\delta^{t-2}+\delta^{t-3}+\ldots+\delta+1\right)\left\|T\left(\rho_{n}\right)-\rho_{n}\right\|+\left\|\rho_{n}-m_{n}\right\| .
\end{aligned}
$$

Furthermore, if we write inequalities (15) under the inequality (12), then we have

$$
\begin{aligned}
\left\|\rho_{n+1}-m_{n+1}\right\| \leq & \left(1-\varsigma_{n}(1-\delta)\right)\left\|\rho_{n}-m_{n}\right\| \\
& +\left(\delta^{m-1}+\delta^{m-2}+\delta^{m-3}+\ldots+\delta^{2}+\delta+1\right)\left(1-\varsigma_{n}(1-\delta)\right)\left\|T \omega_{n}-\omega_{n}\right\| \\
& +\left(1-\varsigma_{n}(1-\delta)\right)\left[\begin{array}{c}
\left.\left[\left(\delta^{p-1}+\delta^{p-2}+\delta^{p-3}+\ldots+\delta\right)\left(1-\tau_{n}(1-\delta)\right)+\left(\delta \tau_{n}+1\right)\right]\left\|T \varkappa_{n}-\varkappa_{n}\right\|\right] \\
+\left(\delta^{t-1}+\delta^{t-2}+\delta^{t-3}+\ldots+\delta+1\right)\left\|T\left(\rho_{n}\right)-\rho_{n}\right\| .
\end{array}\right]
\end{aligned}
$$

Since $\left\{\varsigma_{n}\right\}_{n=0}^{\infty},\left\{\tau_{n}\right\}_{n=0}^{\infty} \subset[0,1]$, we have

$$
\left(1-\varsigma_{n}(1-\delta)\right)<1
$$


NTMSCI 8, No. 1, 71-85 (2020) / www.ntmsci.com

BISK

77

If the notations in the Lemma 2 are defined as follows:

$$
\begin{aligned}
& \varepsilon_{n}=\varsigma_{n}(1-\delta) \\
& a_{n}=\left\|\rho_{n}-m_{n}\right\|
\end{aligned}
$$

and

$$
\begin{aligned}
b_{n}= & \left(\delta^{m-1}+\delta^{m-2}+\delta^{m-3}+\ldots+\delta^{2}+\delta+1\right)\left(1-\varsigma_{n}(1-\delta)\right)\left\|T \omega_{n}-\omega_{n}\right\| \\
& +\left(1-\varsigma_{n}(1-\delta)\right)\left[\begin{array}{c}
{\left[\left(\delta^{p-1}+\delta^{p-2}+\delta^{p-3}+\ldots+\delta\right)\left(1-\tau_{n}(1-\delta)\right)+\left(\delta \tau_{n}+1\right)\right]\left\|T \varkappa_{n}-\varkappa_{n}\right\|} \\
+\left(\delta^{t-1}+\delta^{t-2}+\delta^{t-3}+\ldots+\delta+1\right)\left\|T\left(\rho_{n}\right)-\rho_{n}\right\| .
\end{array}\right]
\end{aligned}
$$

Owing to the facts that $\lim _{n \rightarrow \infty} \rho_{n}=\rho^{*}$ and $T \rho^{*}=\rho^{*}$, we also obtain

$$
\lim _{n \rightarrow \infty}\left\|T \varkappa_{n}-\varkappa_{n}\right\|=\lim _{n \rightarrow \infty}\left\|T \omega_{n}-\omega_{n}\right\|=\lim _{n \rightarrow \infty}\left\|T\left(\rho_{n}\right)-\rho_{n}\right\|=0
$$

Then, it yields $\lim _{n \rightarrow \infty} \frac{b_{n}}{\varepsilon_{n}}=0$ as $n \rightarrow \infty$. Since all the conditions provided, Lemma 2 is applicable. Thus, we get

$$
\lim _{n \rightarrow \infty}\left\|\rho_{n}-m_{n}\right\|=0
$$

If triangular inequality is applied to $\left\|m_{n}-\rho^{*}\right\|$, then we have

$$
\left\|m_{n}-\rho^{*}\right\|=\left\|m_{n}-\rho_{n}+\rho_{n}-\rho^{*}\right\| \leq\left\|m_{n}-\rho_{n}\right\|+\left\|\rho_{n}-\rho^{*}\right\| \rightarrow 0 \text { as } n \rightarrow \infty
$$

Hence, it is concluded from (17) that $\lim _{n \rightarrow \infty}\left\|m_{n}-\rho^{*}\right\|=0$. That is, the Mann iterative process (2) converges to the fixed point $\rho^{*}$ of $T$.

$(i i) \Rightarrow(i):$ Let $m_{n} \rightarrow \rho^{*}$ as $n \rightarrow \infty$. Then, will be proved that the iterative process $\left\{\varsigma_{n}\right\}_{n=0}^{\infty}$ is convergence to $\rho^{*}$.

By using iterative processes (7), (2) and the contraction mapping (1) we have

$$
\begin{aligned}
\left\|m_{n+1}-\rho_{n+1}\right\| & =\left\|\left(1-\varsigma_{n}\right) m_{n}+\varsigma_{n} T m_{n}-\left(\prod_{i=1}^{m} T\right)\left(\left(1-\varsigma_{n}\right) \omega_{n}+\varsigma_{n} T\left(\omega_{n}\right)\right)\right\| \\
& \leq\left(1-\varsigma_{n}\right)\left\|m_{n}-\left(\prod_{i=1}^{m} T\right)\left(\left(1-\varsigma_{n}\right) \omega_{n}+\varsigma_{n} T\left(\omega_{n}\right)\right)\right\|+\varsigma_{n}\left\|T m_{n}-\left(\prod_{i=1}^{m} T\right)\left(\left(1-\varsigma_{n}\right) \omega_{n}+\varsigma_{n} T\left(\omega_{n}\right)\right)\right\| \\
& \leq\left(1-\varsigma_{n}\right)\left\|m_{n}-\prod_{i=1}^{m} T m_{n}+\prod_{i=1}^{m} T m_{n}-\left(\prod_{i=1}^{m} T\right)\left(\left(1-\varsigma_{n}\right) \omega_{n}+\varsigma_{n} T\left(\omega_{n}\right)\right)\right\| \\
& +\varsigma_{n} \delta\left\|m_{n}-\prod_{i=1}^{m-1} T m_{n}+\prod_{i=1}^{m-1} T m_{n}-\left(\prod_{i=1}^{m-1} T\right)\left(\left(1-\varsigma_{n}\right) \omega_{n}+\varsigma_{n} T\left(\omega_{n}\right)\right)\right\| \\
& \leq\left(1-\varsigma_{n}\right)\left\|m_{n}-\prod_{i=1}^{m} T m_{n}\right\|+\left(1-\varsigma_{n}\right)\left\|\prod_{i=1}^{m} T m_{n}-\left(\prod_{i=1}^{m} T\right)\left(\left(1-\varsigma_{n}\right) \omega_{n}+\varsigma_{n} T\left(\omega_{n}\right)\right)\right\|+\varsigma_{n} \delta\left\|m_{n}-\prod_{i=1}^{m-1} T m_{n}\right\| \\
& +\varsigma_{n} \delta\left\|\prod_{i=1}^{m-1} T m_{n}-\left(\prod_{i=1}^{m-1} T\right)\left(\left(1-\varsigma_{n}\right) \omega_{n}+\varsigma_{n} T\left(\omega_{n}\right)\right)\right\| \\
& \leq\left(1-\varsigma_{n}\right)\left\|m_{n}-\prod_{i=1}^{m-1} T m_{n}\right\|+\delta^{m-1}\left\|m_{n}-T m_{n}\right\|+\varsigma_{n} \delta\left\|m_{n}-\prod_{i=1}^{m-2} T m_{n}\right\| \\
& +\delta^{m}\left(1-\varsigma_{n}\right)\left\|m_{n}-\omega_{n}\right\|+\varsigma_{n} \delta^{m}\left\|m_{n}-T\left(\omega_{n}\right)\right\|
\end{aligned}
$$

(C) 2020 BISKA Bilisim Technology 


$$
\begin{aligned}
& \leq\left(1-\varsigma_{n}(1-\delta)\right)\left\|m_{n}-\prod_{i=1}^{m-2} T m_{n}\right\|+\left(\delta^{m-2}+\delta^{m-1}\right)\left\|m_{n}-T m_{n}\right\|+\delta^{m}\left(1-\varsigma_{n}\right)\left\|m_{n}-\omega_{n}\right\|+\varsigma_{n} \delta^{m}\left\|m_{n}-T\left(\omega_{n}\right)\right\| \\
& \leq\left(\delta^{m}+\delta^{m-1}+\delta^{m-2}+\ldots+\delta+\left(1-\varsigma_{n}(1-\delta)\right)\right)\left\|m_{n}-T m_{n}\right\|+\delta^{m}\left(1-\varsigma_{n}(1-\delta)\right)\left\|m_{n}-\omega_{n}\right\| .
\end{aligned}
$$

Again, from iterative processes (7), (2) and the contraction mapping (1) we have

$$
\begin{aligned}
\left\|m_{n}-\omega_{n}\right\| & =\left\|m_{n}-\prod_{j=1}^{p} T m_{n}+\prod_{j=1}^{p} T m_{n}-\left(\prod_{j=1}^{p} T\right)\left(\left(1-\tau_{n}\right) \varkappa_{n}+\tau_{n} T\left(\varkappa_{n}\right)\right)\right\| \\
& \leq\left\|m_{n}-\prod_{j=1}^{p} T m_{n}\right\|+\left\|\prod_{j=1}^{p} T m_{n}-\left(\prod_{j=1}^{p} T\right)\left(\left(1-\tau_{n}\right) \varkappa_{n}+\tau_{n} T\left(\varkappa_{n}\right)\right)\right\| \\
& \leq\left(\tau_{n} \boldsymbol{\delta}^{p}+\boldsymbol{\delta}^{p-1}+\boldsymbol{\delta}^{p-2}+\ldots+\delta\right)\left\|m_{n}-T m_{n}\right\|+\boldsymbol{\delta}^{p}\left(1-\tau_{n}(1-\delta)\right)\left\|m_{n}-\varkappa_{n}\right\| .
\end{aligned}
$$

Similarly, we obtain

$$
\begin{aligned}
\left\|m_{n}-\varkappa_{n}\right\| & =\left\|m_{n}-\prod_{k=1}^{t} T m_{n}+\prod_{k=1}^{t} T m_{n}-\prod_{k=1}^{t} T\left(\rho_{n}\right)\right\| \\
& \leq\left\|m_{n}-\prod_{k=1}^{t} T m_{n}\right\|+\left\|\prod_{k=1}^{t} T m_{n}-\prod_{k=1}^{t} T\left(\rho_{n}\right)\right\| \\
& \leq\left(\delta^{p-1}+\delta^{p-2}+\ldots+\delta\right)\left\|m_{n}-T m_{n}\right\|+\delta^{t}\left\|m_{n}-\rho_{n}\right\| .
\end{aligned}
$$

Furthermore, if we write inequalities (20) and (19) under the inequality (18), then we have

$$
\begin{aligned}
\left\|m_{n+1}-\rho_{n+1}\right\| & \leq\left(\delta^{m}+\delta^{m-1}+\delta^{m-2}+\ldots+\delta+\left(1-\varsigma_{n}(1-\delta)\right)\right)\left\|m_{n}-T m_{n}\right\|+\delta^{m}\left(1-\varsigma_{n}(1-\delta)\right) \\
& \times\left[\left(\tau_{n} \delta^{p}+\delta^{p-1}+\delta^{p-2}+\ldots+\delta\right)\left\|m_{n}-T m_{n}\right\|\right]+\delta^{m+p}\left(1-\varsigma_{n}(1-\delta)\right) \\
& \times\left(1-\tau_{n}(1-\delta)\right)\left[\left(\delta^{p-1}+\delta^{p-2}+\ldots+\delta\right)\left\|m_{n}-T m_{n}\right\|\right] \\
& +\delta^{m+p+t}\left(1-\varsigma_{n}(1-\delta)\right)\left(1-\tau_{n}(1-\delta)\right)\left\|m_{n}-\rho_{n}\right\|
\end{aligned}
$$

or

$$
\begin{aligned}
\left\|m_{n+1}-\rho_{n+1}\right\| \leq & \delta^{m+p+t}\left(1-\varsigma_{n}(1-\delta)\right)\left(1-\tau_{n}(1-\delta)\right)\left\|m_{n}-\rho_{n}\right\| \\
& +\left(\begin{array}{c}
\delta^{m}+\delta^{m-1}+\delta^{m-2}+\ldots+\delta+\left(1-\varsigma_{n}(1-\delta)\right) \\
+\delta^{m}\left(1-\varsigma_{n}(1-\delta)\right) \times\left(\tau_{n} \delta^{p}+\delta^{p-1}+\delta^{p-2}+\ldots+\delta\right) \\
+\delta^{m+p}\left(1-\varsigma_{n}(1-\delta)\right)\left(1-\tau_{n}(1-\delta)\right) \times\left(\delta^{p-1}+\delta^{p-2}+\ldots+\delta\right)
\end{array}\right)\left\|m_{n}-T m_{n}\right\|
\end{aligned}
$$

if the notations in the Lemma 2 are defined as follows:

$$
\begin{aligned}
& \varepsilon_{n}=\varsigma_{n}(1-\delta) \\
& a_{n}=\left\|m_{n}-\rho_{n}\right\|
\end{aligned}
$$

and

$$
b_{n}=\left(\begin{array}{c}
\delta^{m}+\delta^{m-1}+\delta^{m-2}+\ldots+\delta+\left(1-\varsigma_{n}(1-\delta)\right) \\
+\delta^{m}\left(1-\varsigma_{n}(1-\delta)\right) \times\left(\tau_{n} \delta^{p}+\delta^{p-1}+\delta^{p-2}+\ldots+\delta\right) \\
+\delta^{m+p}\left(1-\varsigma_{n}(1-\delta)\right)\left(1-\tau_{n}(1-\delta)\right) \times\left(\delta^{p-1}+\delta^{p-2}+\ldots+\delta\right)
\end{array}\right)\left\|m_{n}-T m_{n}\right\|
$$


Due to the facts that $\lim _{n \rightarrow \infty} m_{n}=\rho^{*}$ and $T \rho^{*}=\rho^{*}$, we also obtain

$$
\lim _{n \rightarrow \infty}\left\|T m_{n}-m_{n}\right\|=0
$$

Then, it yields $\lim _{n \rightarrow \infty} \frac{b_{n}}{\varepsilon_{n}}=0$ as $n \rightarrow \infty$. Since all the conditions of Lemma 2 provided, Lemma 2 is applicable. Thus, we get

$$
\lim _{n \rightarrow \infty}\left\|m_{n}-\rho_{n}\right\|=0 .
$$

If triangular inequality is applied to $\left\|\rho_{n}-\rho^{*}\right\|$, then we have

$$
\begin{aligned}
\left\|\rho_{n}-\rho^{*}\right\| & =\left\|\rho_{n}-m_{n}+m_{n}-\rho^{*}\right\| \\
& \leq\left\|\rho_{n}-m_{n}\right\|+\left\|m_{n}-\rho^{*}\right\| \rightarrow 0
\end{aligned}
$$

Hence, it is concluded from (22) that $\lim _{n \rightarrow \infty}\left\|\rho_{n}-\rho^{*}\right\|=0$. That is, the iterative process (7) converges to the fixed point $\rho^{*}$ of $T$.

Corollary 1. Let $B$ be a Banach space, $E$ be a nonempty, closed, convex subset of $B$ and $T: E \rightarrow E$ be a contraction mapping satisfying condition (1) with a fixed point $\rho^{*}$. If the initial point is the same for all iterations, then the following assertions are require each other:

(1) S-Picard iterative process (3) converges to the fixed point $\rho^{*}$ of $T$,

(2) Vatan two-steps iterative process (4) converges to the fixed point $\rho^{*}$ of $T$,

(3) Thakur iterative process (5) converges to the fixed point $\rho^{*}$ of $T$,

(4) Ishikawa iterative process [6] converges to the fixed point $\rho^{*}$ of $T$,

(5) $S^{*}$ iterative process [17] converges to the fixed point $\rho^{*}$ of $T$,

(6) Mann iterative process (2) converges to the fixed point $\rho^{*}$ of $T$,

(7) Noor iterative process [7] converges to the fixed point $\rho^{*}$ of $T$,

(8) SP iterative process [12] converges to the fixed point $\rho^{*}$ of $T$,

(9) VKF iterative process [14] converges to the fixed point $\rho^{*}$ of $T$,

(10) AK iterative process (6) converges to the fixed point $\rho^{*}$ of $T$,

(11) Picard-Mann iterative process [16] converges to the fixed point $\rho^{*}$ of $T$

(12) Picard-S iterative process [18] converges to the fixed point $\rho^{*}$ of $T$.

Theorem 4. Let $E$ be a nonempty closed convex subset of a Banach space $B$ and $T: E \rightarrow E$ be a contraction mapping. Suppose that $\left\{\rho_{n}\right\}_{n=0}^{\infty}(7),\left\{k_{n}\right\}_{n=0}^{\infty}(3),\left\{v_{n}\right\}_{n=0}^{\infty}(4),\left\{u_{n}\right\}_{n=0}^{\infty}(5)$ and $\left\{x_{n}\right\}_{n=0}^{\infty}(6)$ are five iterative sequences having the same fixed point $\rho^{*}$ and initial condition such that $\left\{\varsigma_{n}\right\}_{n=0}^{\infty},\left\{\tau_{n}\right\}_{n=0}^{\infty} \subset[0,1]$ satisfying $\sum_{n=0}^{\infty} \tau_{i} \varsigma_{i}=\infty, 0<\tau \leq \tau_{n}<1$ and $0<\varsigma \leq \varsigma_{n}<1$, for all $n \in \mathbb{N}$. In which case, the iterative sequence $\left\{\rho_{n}\right\}_{n=0}^{\infty}$ (7) converges faster than the all of $\left\{k_{n}\right\}_{n=0}^{\infty}$ (3), $\left\{v_{n}\right\}_{n=0}^{\infty}$ (4), $\left\{u_{n}\right\}_{n=0}^{\infty}$ (5) and $\left\{x_{n}\right\}_{n=0}^{\infty}$ (6) iterative processes, respectively.

Proof. From the definition of the iteration process (7), we have

$$
\left\|\rho_{n+1}-\rho^{*}\right\| \leq\left(\delta^{m+p+t}\right)^{n+1} \prod_{i=0}^{n}\left(1-\tau_{i}(1-\delta)\right)\left\|\rho_{0}-\rho^{*}\right\| .
$$

From iterative method (6) which is converge to unique fixed point $\rho^{*}$ (see [24], Theorem 2), we have

$$
\left\|x_{n+1}-\rho^{*}\right\| \leq \delta^{3(n+1)} \prod_{i=0}^{n}\left[1-\tau_{i}(1-\delta)\right]\left\|x_{0}-\rho^{*}\right\| .
$$


Taking into account the facts that $\tau \leq \tau_{n}<1$ and $\varsigma \leq \varsigma_{n}<1$, we get

$$
\begin{aligned}
\left\|\rho_{n+1}-\rho^{*}\right\| & \leq\left(\delta^{m+p+t}\right)^{n+1} \prod_{i=0}^{n}\left(1-\tau_{i}(1-\delta)\right)\left\|\rho_{0}-\rho^{*}\right\| \\
& =\left(\delta^{m+p+t}\right)^{n+1}[(1-\tau(1-\delta))]^{(n+1)}\left\|\rho_{0}-\rho^{*}\right\|
\end{aligned}
$$

and

$$
\begin{aligned}
\left\|x_{n+1}-\rho^{*}\right\| & \leq \delta^{3(n+1)} \prod_{i=0}^{n}\left[1-\tau_{i}(1-\delta)\right]\left\|x_{0}-\rho^{*}\right\| \\
& =\delta^{3(n+1)}[1-\tau(1-\delta)]^{(n+1)}\left\|x_{0}-\rho^{*}\right\| .
\end{aligned}
$$

Define

$$
a_{n}=\left(\delta^{m+p+t}\right)^{n+1}[(1-\tau(1-\delta))]^{(n+1)}\left\|\rho_{0}-\rho^{*}\right\|
$$

and

$$
b_{n}=\delta^{3(n+1)}[1-\tau(1-\delta)]^{(n+1)}\left\|x_{0}-\rho^{*}\right\|
$$

then

$$
\Phi_{n}=\frac{a_{n}}{b_{n}}=\frac{\left(\delta^{m+p+t}\right)^{n+1}[(1-\tau(1-\delta))]^{(n+1)}\left\|\rho_{0}-\rho^{*}\right\|}{\delta^{3(n+1)}[1-\tau(1-\delta)]^{(n+1)}\left\|x_{0}-\rho^{*}\right\|}=\left(\delta^{m+p+t-3}\right)^{(n+1)}
$$

By using the ratio test, we have

$$
\lim _{n \rightarrow \infty} \frac{\Phi_{n+1}}{\Phi_{n}}=\lim _{n \rightarrow \infty} \frac{\left(\delta^{m+p+t-3}\right)^{(n+2)}}{\left(\delta^{m+p+t-3}\right)^{(n+1)}}=\delta^{m+p+t-3}<1
$$

which implies that $\sum_{n=0}^{\infty} \Phi_{n}<\infty$. Then, we have

$$
\lim _{n \rightarrow \infty} \frac{\left\|\rho_{n+1}-\rho^{*}\right\|}{\left\|x_{n+1}-\rho^{*}\right\|}=\lim _{n \rightarrow \infty} \frac{a_{n}}{b_{n}}=\lim _{n \rightarrow \infty} \Phi_{n}=0
$$

it yields that the iterative scheme $\left\{\rho_{n}\right\}_{n=0}^{\infty}$ is faster than the iterative scheme $\left\{x_{n}\right\}_{n=0}^{\infty}$. Since the iterative scheme $\left\{x_{n}\right\}_{n=0}^{\infty}$ converges faster than the iterative processes $\left\{k_{n}\right\}_{n=0}^{\infty}(3),\left\{v_{n}\right\}_{n=0}^{\infty}(4)$, and $\left\{u_{n}\right\}_{n=0}^{\infty}(5)$, the new iterative method $\left\{\rho_{n}\right\}_{n=0}^{\infty}$ is even faster than them. Moreover, for $m, p, t=1$, the new iterative method $\left\{\rho_{n}\right\}_{n=0}^{\infty}$ reduces to

$$
\left\{\begin{array}{l}
\rho_{0} \in C \text { initial condition } \\
s_{n+1}=T\left(\left(1-\varsigma_{n}\right) r_{n}+\varsigma_{n} T\left(r_{n}\right)\right) \\
r_{n}=T\left(\left(1-\tau_{n}\right) \varkappa_{n}+\tau_{n} T\left(\varkappa_{n}\right)\right) \\
\varkappa_{n}=T\left(s_{n}\right), \text { for all } n \in \mathbb{N} .
\end{array}\right.
$$

Hence, we also get

$$
\left\|s_{n+1}-\rho^{*}\right\| \leq\left(\delta^{1+1+1}\right)^{n+1} \prod_{i=0}^{n}\left[1-\tau_{i}(1-\delta)\right]\left\|\rho_{0}-\rho^{*}\right\|=\left(\delta^{3}\right)^{n+1} \prod_{i=0}^{n}\left[1-\tau_{i}(1-\delta)\right]\left\|\rho_{0}-\rho^{*}\right\| .
$$

If the processes are repeated under conditions mentioned above, it is easy to see that the iterative schemes (24) and (6) converge to the operator's fixed point with equal convergence speed. 
Now, we will show that the correctness of the rate of convergence by some examples.

Example 1. For initial point $x_{0}=0.5$, we consider the non-linear quation $f(x)=x^{3}+4 x^{2}-10=0$. If this equation is written in the form of fixed point method, then we have $T x=(10 /(4+x))^{1 / 2}$. The numerical solution of this equation is 1,365230013414100 for fifteen decimal. The following table and graphs show the correctness of the solution.

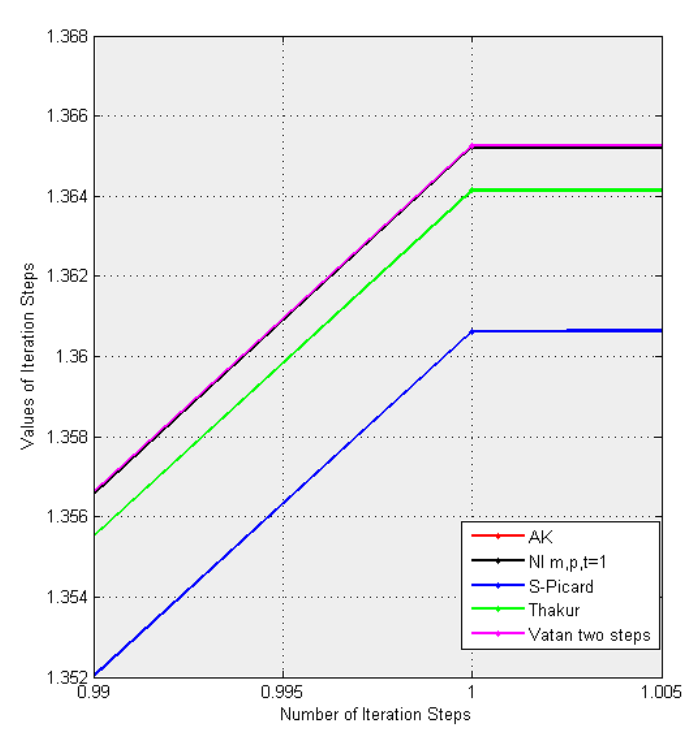

Fig. 1: The convergence rate comparison of fixed point iteration methods AK, NI ( $\mathrm{m}, \mathrm{p}, \mathrm{t}=1)$, S-Picard, Thakur and Vatan two-steps for the solution of $f(x)=x^{3}+4 x^{2}-10=0$ nonlinear equation.

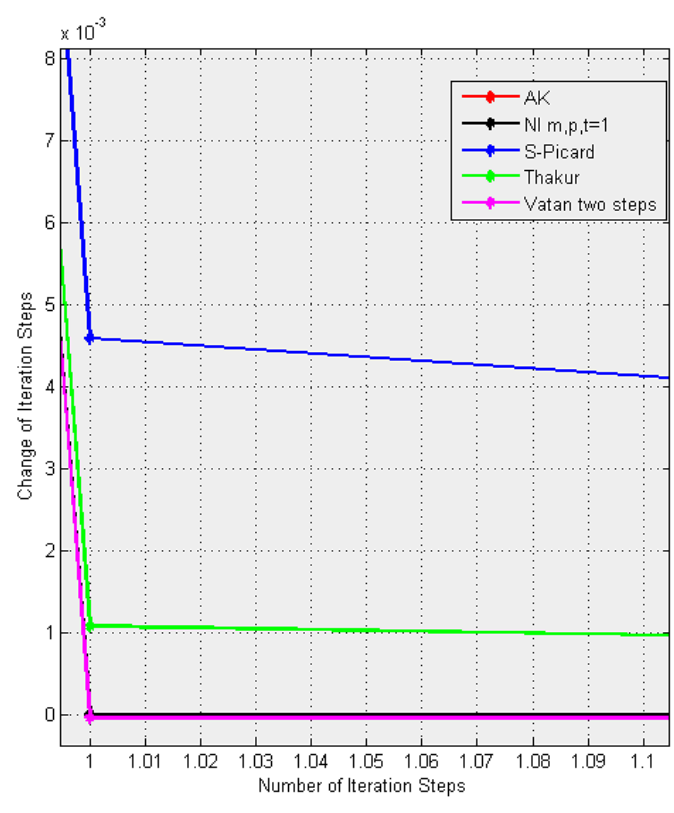

Fig. 2: The derivative comparison of fixed point iteration methods AK, NI ( $\mathrm{m}, \mathrm{p}, \mathrm{t}=1)$, S-Picard, Thakur and Vatan two-steps for the solution of $f(x)=x^{3}+4 x^{2}-10=0$ nonlinear equation.

Table 1: Comparison of fixed point iteration methods for the solution of the nonlinear equation given in Example 1

\begin{tabular}{cccccc}
\hline Iteration & $\mathbf{A K}$ & $\mathbf{N I} \mathbf{m , p , t = 1}$ & $\mathbf{S - P i c a r d}$ & Thakur & Vatan two steps \\
\hline$x_{0}$ & 0,5 & 0,5 & 0,5 & 0,5 & 0,5 \\
$x_{1}$ & $1,3652 \ldots 8670$ & $1,3652 \ldots 7280$ & $1,3606 \ldots 9320$ & $1,3641 \ldots 5350$ & $1,3652 \ldots 4140$ \\
$x_{2}$ & $1,3652 \ldots 1780$ & $1,3652 \ldots 4640$ & $1,3652 \ldots 8780$ & $1,3652 \ldots 502920$ & $1,3652 \ldots 7650$ \\
$x_{3}$ & $1,3652 \ldots 4100$ & $1,3652 \ldots 4100$ & $1,3652 \ldots 6050$ & $1,3652 \ldots 1310$ & $1,3652 \ldots 4200$ \\
$x_{4}$ & $1,3652 \ldots 4100$ & $1,3652 \ldots 4100$ & $1,3652 \ldots 7600$ & $1,3652 \ldots 2090$ & $1,3652 \ldots 4100$ \\
$\vdots$ & $\vdots$ & $\vdots$ & $\vdots$ & $\vdots$ & $\vdots$ \\
$x_{6}$ & $1,3652 \ldots 4100$ & $1,3652 \ldots 4100$ & $1,3652 \ldots 4090$ & $1,3652 \ldots 4100$ & $1,3652 \ldots 4100$ \\
$x_{7}$ & $1,3652 \ldots 4100$ & $1,3652 \ldots 4100$ & $1,3652 \ldots 4100$ & $1,3652 \ldots 4100$ & $1,3652 \ldots 4100$ \\
\hline
\end{tabular}

Example 2. For initial point $x_{0}=0.1$, we consider the non-lineare quation $f(x)=x^{3}+5 x-5=0$. If this equation is written in the form of fixed point method, then we have $T x=\left(5-x^{3}\right) / 5$. The numerical solution of this equation is 0,868830020341475 for fifteen decimal. The following table and graphs show the correctness of the solution.

Theorem 5. Let $E$ be a nonempty closed convex subset of a Banach space $B$ and $T, \check{T}: E \rightarrow E$ be two contraction mappings such that $\check{T}$ is an approximate operator of $T$. Suppose that $\left\{\rho_{n}\right\}_{n=0}^{\infty}$ and $\left\{\widehat{\rho}_{n}\right\}_{n=0}^{\infty}$ are two iterative sequences, 


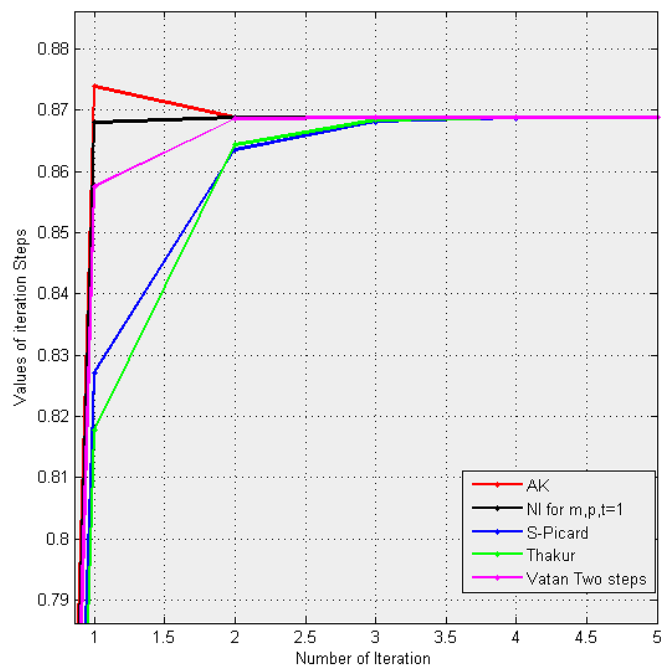

Fig. 3: The convergence rate comparison of fixed point iteration methods $\mathrm{AK}, \mathrm{NI}(\mathrm{m}, \mathrm{p}, \mathrm{t}=1)$, S-Picard, Thakur and Vatan two-steps for the solution of $f(x)=x^{3}+5 x-5=0$ nonlinear equation.

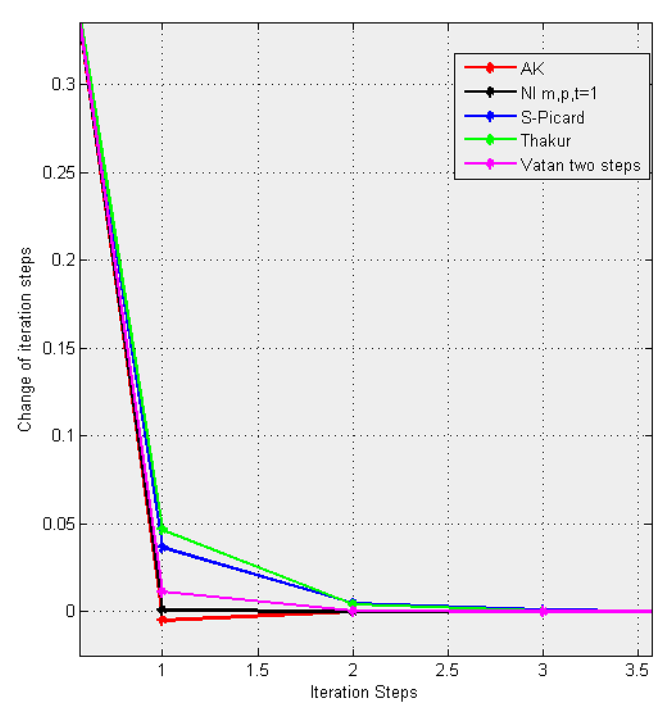

Fig. 4: The derivative comparison of fixed point iteration methods AK, NI ( $\mathrm{m}, \mathrm{p}, \mathrm{t}=1)$, S-Picard, Thakur and Vatan two-steps for the solution of $f(x)=x^{3}+4 x^{2}-10=0$ nonlinear equation.

Table 2: Comparison of fixed point iteration methods for the solution of the nonlinear equation given in Example 1

\begin{tabular}{cccccc}
\hline Iteration & $\mathbf{A K}$ & $\mathbf{N I} \mathbf{~ m , p , t = 1}$ & $\mathbf{S - P i c a r d}$ & Thakur & Vatan two steps \\
\hline$x_{0}$ & 0,1 & 0,1 & 0,1 & 0,1 & 0,1 \\
$x_{1}$ & $0,8738 \ldots 3874$ & $0,8680 \ldots 6244$ & $0,8271 \ldots 4353$ & $0,8176 \ldots 9455$ & $0,8575 \ldots 7045$ \\
$\vdots$ & $\vdots$ & $\vdots$ & $\vdots$ & $\vdots$ & $\vdots$ \\
$x_{6}$ & $0,8688 \ldots 1393$ & $0,8688 \ldots 1475$ & $0,8688 \ldots 9513$ & $0,8688 \ldots 4038$ & $0,8688 \ldots 1818$ \\
$x_{7}$ & $0,8688 \ldots 1475$ & $0,8688 \ldots 1475$ & $0,8688 \ldots 9117$ & $0,8688 \ldots 8736$ & $0,8688 \ldots 1327$ \\
$\vdots$ & $\vdots$ & $\vdots$ & $\vdots$ & $\vdots$ & $\vdots$ \\
$x_{9}$ & $0,8688 \ldots 1475$ & $0,8688 \ldots 1475$ & $0,8688 \ldots 6972$ & $0,8688 \ldots 8355$ & $0,8688 \ldots 1475$ \\
$\vdots$ & $\vdots$ & $\vdots$ & $\vdots$ & $\vdots$ & $\vdots$ \\
$x_{15}$ & $0,8688 \ldots 1475$ & $0,8688 \ldots 1475$ & $0,8688 \ldots 6972$ & $0,8688 \ldots 1475$ & $0,8688 \ldots 1475$ \\
$\vdots$ & $\vdots$ & $\vdots$ & $\vdots$ & $\vdots$ & $\vdots$ \\
$x_{19}$ & $0,8688 \ldots 1475$ & $0,8688 \ldots 1475$ & $0,8688 \ldots 1475$ & $0,8688 \ldots 1475$ & $0,8688 \ldots 1475$ \\
\hline
\end{tabular}

which have the fixed points $\rho^{*}$ and $\widehat{\rho}^{*}$, defined by (7) and (25), respectively, such that $\left\{\varsigma_{n}\right\}_{n=0}^{\infty},\left\{\tau_{n}\right\}_{n=0}^{\infty} \subset[0,1]$ and $\varsigma_{n} \geq 1 / 2$ satisfying $\sum_{n=0}^{\infty} \varsigma_{n}=\infty$.

$$
\left\{\begin{array}{l}
\widehat{\rho}_{0} \in C \text { initial condition } \\
\widehat{\rho}_{n+1}=\left(\prod_{i=1}^{m} \check{T}\right)\left(\left(1-\varsigma_{n}\right) \widehat{\omega}_{n}+\varsigma_{n} \check{T}\left(\widehat{\omega}_{n}\right)\right) \\
\widehat{\omega}_{n}=\left(\prod_{j=1}^{p} \check{T}\right)\left(\left(1-\tau_{n}\right) \widehat{\varkappa}_{n}+\tau_{n} \check{T}\left(\widehat{\varkappa}_{n}\right)\right) \\
\widehat{\varkappa}_{n}=\left(\prod_{k=1}^{t} \check{T}\right)\left(\widehat{\rho}_{n}\right), \text { for all } n \in \mathbb{N} .
\end{array}\right.
$$


In which case, the following claim holds:

$$
\left\|\rho^{*}-\widehat{\rho}^{*}\right\| \leq \frac{6 \varepsilon}{1-\delta}
$$

where $\varepsilon$ is a constant in the positive real numbers.

Proof. By using (7) and (25) iterative processes, we have

$$
\begin{aligned}
\left\|\varkappa_{n}-\widehat{\varkappa}_{n}\right\| & =\left\|\left(\prod_{k=1}^{t} T\right)\left(\rho_{n}\right)-\left(\prod_{k=1}^{t} \check{T}\right)\left(\widehat{\rho}_{n}\right)\right\| \\
& \leq\left\|\left(\prod_{k=1}^{t} T\right)\left(\rho_{n}\right)-\left(\prod_{k=1}^{t} T\right)\left(\widehat{\rho}_{n}\right)\right\|+\left\|\left(\prod_{k=1}^{t} T\right)\left(\widehat{\rho}_{n}\right)-\left(\prod_{k=1}^{t} \check{T}\right)\left(\widehat{\rho}_{n}\right)\right\| \\
& \leq \delta^{t}\left\|\rho_{n}-\widehat{\rho}_{n}\right\|+\varepsilon .
\end{aligned}
$$

Considering the inequality (26), we obtain

$$
\begin{aligned}
\left\|\omega_{n}-\widehat{\omega}_{n}\right\| & =\left\|\left(\prod_{j=1}^{p} T\right)\left(\left(1-\tau_{n}\right) \varkappa_{n}+\tau_{n} T\left(\varkappa_{n}\right)\right)-\left(\prod_{j=1}^{p} \check{T}\right)\left(\left(1-\tau_{n}\right) \widehat{\varkappa}_{n}+\tau_{n} \check{T}\left(\widehat{\varkappa}_{n}\right)\right)\right\| \\
& \leq\left\|\left(\prod_{j=1}^{p} T\right)\left(\left(1-\tau_{n}\right) \varkappa_{n}+\tau_{n} T\left(\varkappa_{n}\right)\right)-\left(\prod_{j=1}^{p} T\right)\left(\left(1-\tau_{n}\right) \widehat{\varkappa}_{n}+\tau_{n} \breve{T}\left(\widehat{\varkappa}_{n}\right)\right)\right\| \\
& +\left\|\left(\prod_{j=1}^{p} T\right)\left(\left(1-\tau_{n}\right) \widehat{\varkappa}_{n}+\tau_{n} \breve{T}\left(\widehat{\varkappa}_{n}\right)\right)-\left(\prod_{j=1}^{p} \check{T}\right)\left(\left(1-\tau_{n}\right) \widehat{\varkappa}_{n}+\tau_{n} \check{T}\left(\widehat{\varkappa}_{n}\right)\right)\right\| \\
& \leq \delta^{p}\left\|\left(1-\tau_{n}\right) \varkappa_{n}+\tau_{n} T\left(\varkappa_{n}\right)-\left(1-\tau_{n}\right) \widehat{\varkappa}_{n}-\tau_{n} \check{T}\left(\widehat{\varkappa}_{n}\right)\right\|+\varepsilon \\
& \leq \delta^{p}\left(1-\tau_{n}\right)\left\|\varkappa_{n}-\widehat{\varkappa}_{n}\right\|+\delta^{p} \tau_{n}\left\|T\left(\varkappa_{n}\right)-\check{T}\left(\widehat{\varkappa}_{n}\right)\right\|+\varepsilon \\
& \leq \delta^{p}\left(1-\tau_{n}\right)\left\|\varkappa_{n}-\widehat{\varkappa}_{n}\right\|+\delta^{p} \tau_{n}\left\|T\left(\varkappa_{n}\right)-T\left(\widehat{\varkappa}_{n}\right)\right\|+\delta^{p} \tau_{n}\left\|T\left(\widehat{\varkappa}_{n}\right)-\breve{T}\left(\widehat{\varkappa}_{n}\right)\right\|+\varepsilon \\
& \leq \delta^{p}\left(1-\tau_{n}\right)\left(\delta^{t}\left\|\rho_{n}-\widehat{\rho}_{n}\right\|+\varepsilon\right)+\delta^{p+1} \tau_{n}\left(\delta^{t}\left\|\rho_{n}-\widehat{\rho}_{n}\right\|+\varepsilon\right)+\delta^{p} \tau_{n} \varepsilon+\varepsilon \\
& =\delta^{p+t}\left(1-\tau_{n}(1-\delta)\right)\left\|\rho_{n}-\widehat{\rho}_{n}\right\|+\left(\delta^{p}\left[\left(1-\tau_{n}(1-\delta)\right)+\tau_{n}\right]+1\right) \varepsilon .
\end{aligned}
$$

Similarly, using the inequality (27), we obtain

$$
\begin{aligned}
& \left\|\rho_{n+1}-\widehat{\rho}_{n+1}\right\|=\left\|\left(\prod_{i=1}^{m} T\right)\left(\left(1-\varsigma_{n}\right) \omega_{n}+\varsigma_{n} T\left(\omega_{n}\right)\right)-\left(\prod_{i=1}^{m} \check{T}\right)\left(\left(1-\varsigma_{n}\right) \widehat{\omega}_{n}+\varsigma_{n} \check{T}\left(\widehat{\omega}_{n}\right)\right)\right\| \\
& \leq\left\|\left(\prod_{i=1}^{m} T\right)\left(\left(1-\varsigma_{n}\right) \omega_{n}+\varsigma_{n} T\left(\omega_{n}\right)\right)-\left(\prod_{i=1}^{m} T\right)\left(\left(1-\varsigma_{n}\right) \widehat{\omega}_{n}+\varsigma_{n} \check{T}\left(\widehat{\omega}_{n}\right)\right)\right\| \\
& +\left\|\left(\prod_{i=1}^{m} T\right)\left(\left(1-\varsigma_{n}\right) \widehat{\omega}_{n}+\varsigma_{n} \check{T}\left(\widehat{\omega}_{n}\right)\right)-\left(\prod_{i=1}^{m} \check{T}\right)\left(\left(1-\varsigma_{n}\right) \widehat{\omega}_{n}+\varsigma_{n} \check{T}\left(\widehat{\omega}_{n}\right)\right)\right\| \\
& \leq \delta^{m}\left\|\left(1-\varsigma_{n}\right) \omega_{n}+\varsigma_{n} T\left(\omega_{n}\right)-\left(1-\varsigma_{n}\right) \widehat{\omega}_{n}+\varsigma_{n} \breve{T}\left(\widehat{\omega}_{n}\right)\right\|+\varepsilon \\
& \leq \delta^{m}\left(1-\varsigma_{n}\right)\left\|\omega_{n}-\widehat{\omega}_{n}\right\|+\delta^{m} \varsigma_{n}\left\|T\left(\omega_{n}\right)-\check{T}\left(\widehat{\omega}_{n}\right)\right\|+\varepsilon \\
& \leq \delta^{m}\left(1-\varsigma_{n}\right)\left\|\omega_{n}-\widehat{\omega}_{n}\right\|+\delta^{m} \varsigma_{n}\left\|T\left(\omega_{n}\right)-T\left(\widehat{\omega}_{n}\right)\right\|+\delta^{m} \varsigma_{n}\left\|T\left(\widehat{\omega}_{n}\right)-\check{T}\left(\widehat{\omega}_{n}\right)\right\|+\varepsilon \\
& \leq \delta^{m}\left(1-\varsigma_{n}(1-\delta)\right)\left\|\omega_{n}-\widehat{\omega}_{n}\right\|+\delta^{m} \varsigma_{n} \varepsilon+\varepsilon \\
& \leq \delta^{m}\left(1-\varsigma_{n}(1-\delta)\right)\left[\begin{array}{c}
\delta^{p+t}\left(1-\tau_{n}(1-\delta)\right)\left\|\rho_{n}-\widehat{\rho}_{n}\right\| \\
+\left(\delta^{p}\left[\left(1-\tau_{n}(1-\delta)\right)+\tau_{n}\right]+1\right) \varepsilon
\end{array}\right]+\delta^{m} \varsigma_{n} \varepsilon+\varepsilon \\
& =\delta^{m+p+t}\left(1-\varsigma_{n}(1-\delta)\right)\left(1-\tau_{n}(1-\delta)\right)\left\|\rho_{n}-\widehat{\rho}_{n}\right\|
\end{aligned}
$$




$$
\begin{aligned}
& +\delta^{m}\left(1-\varsigma_{n}(1-\delta)\right)\left(\delta^{p}\left[\left(1-\tau_{n}(1-\delta)\right)+\tau_{n}\right]+1\right) \varepsilon+\delta^{m} \varsigma_{n} \varepsilon+\varepsilon \\
& \leq \delta^{m+p+t}\left(1-\varsigma_{n}(1-\delta)\right)\left(1-\tau_{n}(1-\delta)\right)\left\|\rho_{n}-\widehat{\rho}_{n}\right\|+6 \varsigma_{n}(1-\delta) \frac{\varepsilon}{(1-\delta)}
\end{aligned}
$$

Since $\left\{\varsigma_{n}\right\}_{n=0}^{\infty},\left\{\tau_{n}\right\}_{n=0}^{\infty} \subset[0,1]$ and $\varsigma_{n} \geq \frac{1}{2}$, we have

$$
\left(1-\varsigma_{n}(1-\delta)\right)<1
$$

and

$$
\delta^{m}\left(1-\varsigma_{n}(1-\delta)\right)\left(\delta^{p}\left[\left(1-\tau_{n}(1-\delta)\right)+\tau_{n}\right]+1\right) \varepsilon+\delta^{m} \varsigma_{n} \varepsilon+\varepsilon \leq 6 \varsigma_{n}(1-\delta) \frac{\varepsilon}{(1-\delta)} .
$$

Hence, we obtain

$$
\begin{aligned}
\left\|\rho_{n+1}-\widehat{\rho}_{n+1}\right\| & \leq \delta^{m+p+t}\left(1-\varsigma_{n}(1-\delta)\right)\left(1-\tau_{n}(1-\delta)\right)\left\|\rho_{n}-\widehat{\rho}_{n}\right\|+6 \varsigma_{n}(1-\delta) \frac{\varepsilon}{(1-\delta)} \\
& \leq\left(1-\varsigma_{n}(1-\delta)\right)\left\|\rho_{n}-\widehat{\rho}_{n}\right\|+\varsigma_{n}(1-\delta) \frac{6 \varepsilon}{(1-\delta)}
\end{aligned}
$$

Define

$$
\begin{gathered}
a_{n}=\left\|\rho_{n}-\widehat{\rho}_{n}\right\|, \\
\varepsilon_{n}=\varsigma_{n}(1-\delta)
\end{gathered}
$$

and

$$
b_{n}=\frac{6 \varepsilon}{(1-\delta)}
$$

By Lemma 2 together with the inequality (28), we get

$$
0 \leq \lim _{n \rightarrow \infty} \sup \left\|\rho_{n}-\widehat{\rho}_{n}\right\| \leq \lim _{n \rightarrow \infty} \sup \frac{6 \varepsilon}{(1-\delta)} .
$$

From Theorem 2, we have $\lim _{n \rightarrow \infty} \rho_{n}=\rho^{*}$ and $\lim _{n \rightarrow \infty} \widehat{\rho}_{n}=\widehat{\rho}^{*}$. This implies that

$$
\left\|\rho^{*}-\widehat{\rho}^{*}\right\| \leq \frac{6 \varepsilon}{(1-\delta)}
$$

\section{Competing interests}

The authors declare that they have no competing interests.

\section{Authors' contributions}

All authors have contributed to all parts of the article. All authors read and approved the final manuscript.

\section{References}

[1] Dogan, K. Karakaya, V. On the Convergence and Stability Results for a New General Iterative Process. Scientific world journal 2014, 2014. 
[2] Dogan, K. Karakaya, V. A study in the fixed point theory for a new iterative scheme and a class of generalized mapings.Creat. Math. Inform. 2018, 27, 151-160.

[3] Karakaya, V. Atalan, Y. Dogan, K. and El Houda Bouzara, N. Some fixed point results for a new three steps iteration process in Banach spaces. Fixed Point Theory 2017, 18, 625-640.

[4] Mann, WR. Mean value methods in iterations. Proc. Amer. Math. Soc. 1953, 44, 506-510.

[5] Khan, A. R. Gürsoy, F. Karakaya, V. Jungck-Khan iterative scheme and higher convergence rate. International Journal of Computer Mathematics 2016, 93, 1-14.

[6] Ishikawa, S. Fixed points by a new iteration method. Proc. Amer. Math. Soc. 1974, 44, 147-150.

[7] Noor, M.A. New approximation schemes for general variational inequalities. J. Math. Anal. Appl. 2000, 251, $217-229$.

[8] Rhoades, B. E. Fixed point iterations using infinite matrices. Trans. Am. Math. Soc. 1974, 196, 161-176.

[9] Rhoades, B. E. Comments on two fixed point iteration methods. J. Math. Anal. Appl. 1976, 56, 741-750.

[10] Borwein, D. Borwein, J. Fixed point iterations for real functions. J. Math. Anal. Appl. 1991, 157, 112-126.

[11] Qing, Y. Qihou, L. The necessary and sufficient condition for the convergence of Ishikawa iteration on an arbitrary interval. $J$. Math. Anal. Appl. 2006, 323, 1383-1386.

[12] Phuengrattana, W. Suantai, S. On the rate of convergence of Mann, Ishikawa, Noor and SP-iterations for continuousfunctions on an arbitrary interval. J. Comput. Appl. Math. 2011, 235, 3006-3014.

[13] Agarwal, R.P. O'Regan, D. and Sahu, D.R. Iterative construction of fixed points of nearly asymptotically nonexpansive mappings. J. Nonlinear Convex Anal. 2007, 8, 61-79.

[14] Karakaya, V. Dogan, K. Gursoy, F. and Erturk, M. Fixed point of a new three-step iteration algorithm under contractive-like operators over normed spaces, Abst and Appl Anal. 2013, 2013, 9 pages.

[15] Kadioglu, N. and Yildirim, I. On the convergence of an iteration method for continuous mappings on an arbitrary interval, Fixed Point Theory and A. 2013, 2013, 124.

[16] Khan, S.H. A Picard-Mann hybrid iterative process. Fixed Point Theory and A. 2013, 2013, 69.

[17] Karahan, I. and Ozdemir, M. Fixed point problems of the Picard-Mann hybrid iterative process for continuous functions on an arbitrary interval. Fixed Point Theory and A. 2013, 2013, 244-256.

[18] Gürsoy, F. and Karakaya, V. A Picard-S hybrid type iteration method for solving a differential equation with retarded argument. arXiv preprint arXiv 2014, 1403.2546.

[19] Sainuan, P. Rate of Convergence of P-Iteration and S-Iteration for Continuous Functions on Closed Intervals. Thai Journal of Mathematics 2015, 13, 449-457.

[20] Weng, X. Fixed point iteration for local strictly pseudocontractive mapping. Proc Am Math Soc 1991, 113, 727-731.

[21] Karakaya, V. Atalan, Y. Doğan, K. and Bouzara, NEH. Convergence analysis for a new faster iteration method. Ist. Com.Unv.J.Sci. 2017, 15, 35-53.

[22] Doğan, K. and Karakaya, V. A study in the fixed point theory for a new iterative scheme and a class of generalized mappings. Creat. Math. Inform. 2018, 25, 151 - 160.

[23] Thakur, D. and Postolache, M. A new iterative scheme for numerical reckoning fixed points of Suzuki's generalized nonexpansive mappings. Appl Math Comput 2016, 275, 147-155.

[24] Ullah, K. andArshad, M. On different results for new three step iteration process in Banach spaces. SpringerPlus 2016, 5(1), 1616.

[25] Berinde, V. Picard iteration converges faster than Mann iteration for a class of quasi-contractive operators. Fixed Point Theory A. 2004, 2, 97-105.

[26] S oltuz SM. and Grosan T. Data dependence for Ishikawa iteration when dealing with contractive like operators. Fixed Point Theory A. 2008, 2008, 1-7. 